\title{
Adherence to prescribed antihypertensive drug treatments: longitudinal study of electronically compiled dosing histories
}

\author{
Bernard Vrijens, chief scientist and adjunct professor of biostatistics, ${ }^{1,2}$ Gäbor Vincze, principal health \\ economist , ${ }^{3}$ Paulus Kristanto, senior biostatistician, , John Urquhart, professor of biopharmaceutical \\ sciences ${ }^{4}$ Michel Burnier, professor of nephrology ${ }^{5}$
}

\section{${ }^{1}$ Pharmionic Research Center, Rue des Cyclistes Frontière 24, 4600 Visé, Belgium \\ ${ }^{2}$ University of Liège, Department of Biostatistics and Medical Informatics, Liège, Belgium \\ ${ }^{3}$ Novartis Pharma, Basel, Switzerland \\ ${ }^{4}$ University of California at San Francisco, Center for Drug Development Science, \\ San Francisco, USA \\ ${ }^{5}$ University Hospital of Lausanne, Division of Nephrology and Hypertension Consultation, Lausanne, Switzerland \\ Correspondence to: B Vrijens} bernard.vrijens@pharmionic.com

doi:10.1136/bmj.39553.670231.25

\section{ABSTRACT}

Objective To describe characteristics of dosing history in patients prescribed a once a day antihypertensive medication.

Design Longitudinal database study.

Setting Clinical studies archived in database for 19892006.

Participants Patients who participated in the studies whose dosing histories were available through electronic monitoring.

Main outcome measures Persistence with prescribed antihypertensive treatment and execution of their once a day drug dosing regimens.

Results The database contained dosing histories of 4783 patients with hypertension. The data came from 21 phase IV clinical studies, with lengths ranging from 30 to 330 days and involving 43 different antihypertensive drugs, including angiotensin II receptor blockers $(n=2088)$, calcium channel blockers ( $n=937$ ), angiotensin converting enzyme inhibitors $(n=665), \beta$ blockers $(n=195)$, and diuretics $(n=155)$. About half of the patients who were prescribed an

antihypertensive drug had stopped taking it within one year. On any day, patients who were still engaged with the drug dosing regimen omitted about $10 \%$ of the scheduled doses: $42 \%$ of these omissions were of a single day's dose, whereas $43 \%$ were part of a sequence of several days (three or more days-that is, drug "holidays"). Almost half of the patients had at least one drug holiday a year. The likelihood that a patient would discontinue treatment early was inversely related to the quality of his or her daily execution of the dosing regimen.

Conclusions Early discontinuation of treatment and suboptimal daily execution of the prescribed regimens are the most common facets of poor adherence with once a day antihypertensive drug treatments. The shortfalls in drug exposure that these dosing errors create might be a common cause of low rates of blood pressure control and high variability in responses to prescribed antihypertensive drugs.

\section{INTRODUCTION}

Hypertension is a major risk factor for the development of cardiovascular disease. ${ }^{12}$ Effective and well tolerated once a day antihypertensive drugs are now available. Yet poor adherence with prescribed treatment continues to be one of the main causes of unsatisfactory control of blood pressure and might lead to target organ damage and increased cardiovascular risk. ${ }^{3-5}$ Although many studies have examined the "adherence issue" over many years, the absence of a common taxonomy and the lack of reliable measurements of ambulatory patients' exposure to prescribed pharmaceuticals have resulted in much confusion, with "adherence rates" ranging from $35 \%$ to as high as $97 \%{ }^{67}$

One aspect of the problem is that traditional methods (pill counts, questionnaires, patients' diaries, measurements of drug concentration in plasma, etc) have repeatedly been shown to overestimate adherence. ${ }^{489}$ A reliable assessment of the prevalence of poor adherence, including short persistence, can be inferred over long term follow-up from the timing of refills in large prescription databases, ${ }^{10} 11$ but refill audits do not show when dosing errors occurred, including, most importantly, the exact time when the patient stopped taking the drug. The same limitation applies to measurements of drug concentrations in plasma, which usually do not take into account the fact that patients' drug taking behaviour is a dynamic process that changes over time and is subject to strong bias created by white coat effects that typically increase adherence in the 24-48 hours before a scheduled visit to the clinic or laboratory. ${ }^{12-15}$

A common error that has led to the widespread belief that "overall adherence" in hypertension treatment is only about $50-60 \%{ }^{16}$ is the failure to distinguish between the two major components of a course of ambulatory pharmacotherapy: the quality of execution while the patient is engaged with his or her dosing regimen; and early discontinuation - that is, disengagement from his or her dosing regimen, known as "short persistence." The distinction between these two aspects of the patients' adherence to a prescribed regimen is crucial because the dynamics as well as the clinical and economic consequences of poor quality of execution and short persistence can differ markedly. 
We characterised the most common dosing errors observed in a large group of patients with hypertension who were prescribed a once a day antihypertensive treatment.

\section{METHODS}

Study design and setting

We carried out a longitudinal study of patients' adherence to their once a day antihypertensive medications on the basis of dosing histories that have been electronically compiled by a medication event monitor (MEMS, Aardex, Zug, Switzerland) during phase IV clinical studies in 1989-2006. The monitors automatically record the date and time of each opening of the medication container. ${ }^{17}$ The dosing histories were archived in the Pharmionic Knowledge Centre (PKC) database, on to which researchers entered suitably anonymised data on dosing history. The owners of each dataset gave consent for the use of these data for retrospective analyses. The database currently contains data on more than 20000 ambulatory patients whose dosing histories have been electronically compiled during clinical studies of various lengths in ambulatory pharmacotherapy (cardiovascular, central nervous systems, infections, gastrointestinal, endocrine, etc). Reliable assessment of patients' adherence was a secondary objective in the included studies, which varied somewhat in primary objectives. Our study thus constitutes secondary use of the data. The database provides the means to identify the most common dosing errors in the pharmacotherapy of various diseases.

\section{Patients}

We included all patients who participated in clinical studies involving once a day antihypertensive drug
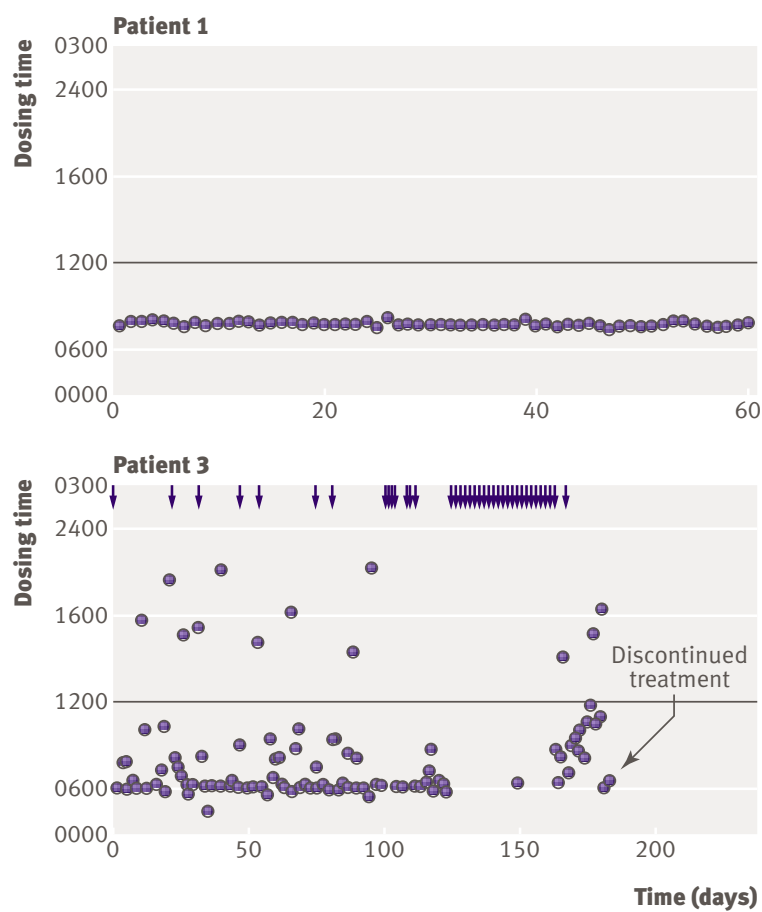

treatments during 1989-2006 found in the Pharmionic Knowledge Centre database. Nearly all patients in the database were prescribed relatively recently introduced pharmaceuticals. Dosing histories of all patients in the studies were automatically captured with an electronic medication event monitor, a container with a threaded closure with electronic time stamping microcircuitry. The prescribed antihypertensive medications were given to the patients in the monitored container with the instruction to open it when it was time to take the medicine, to remove and take the prescribed dose, then promptly close the package. Each patient gave informed consent regarding the use of the container.

\section{Definitions}

We defined adherence (or compliance) as a dimensionless, blanket term encompassing the extent to which patients' drug dosing histories conform, or not, to their corresponding prescribed drug dosing regimen. Adherence can be broken into two main components $^{18}$ : persistence is the length of time during which the medication is taken - that is, the time from the first taken dose to the last taken dose; and execution is the multidimensional outcome of the comparison of two time series: the prescribed drug dosing regimen and the patient's drug dosing history while he or she is still engaged with treatment.

\section{Statistical methods}

Each individual's dosing history can be visualised by using a chronology plot that displays the time of each putative dose (each opening and closing of the monitored container) on a scatter plot of time of opening (24 hour clock) and calendar date. The

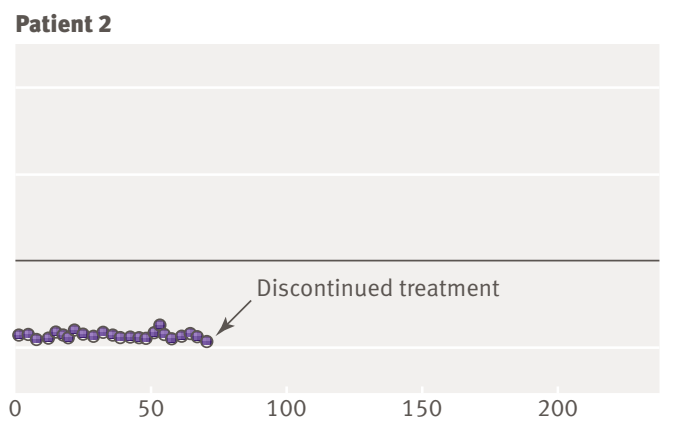

Patient 4

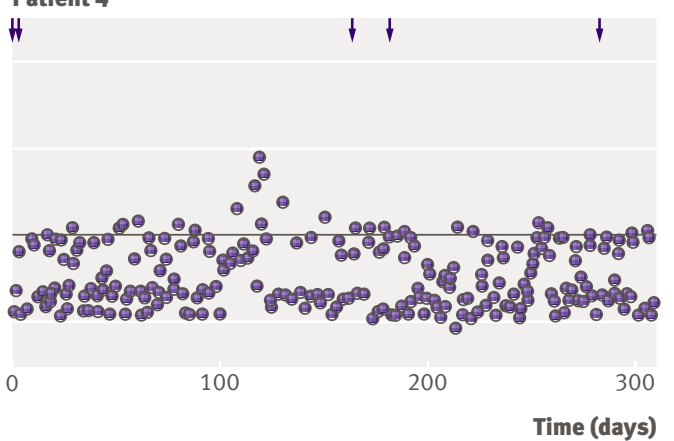




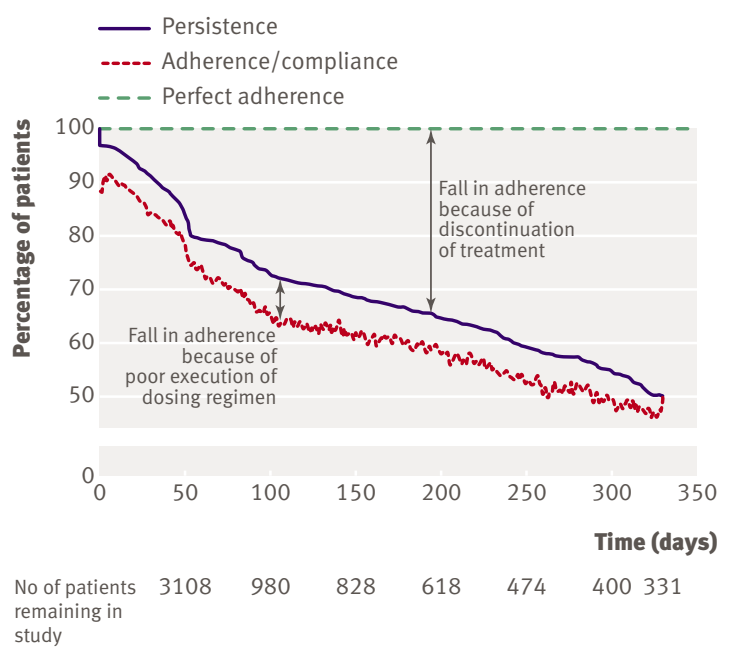

Fig 2 | Time course of adherence/compliance parameters (execution, persistence)

chronology plot shows, for each patient separately, the different aspects of a patient's dosing history: nonpersistence, variability in time of drug intake, delayed doses, single or sequentially missed multiple dosesthat is, drug holidays, and extra doses.

Summarising this multidimensional information across a population is a challenge and depends in part on whether one takes the perspective of a group or an individual patient. ${ }^{19}$ One salient aspect of a group's adherence can be described by plotting the proportion of patients who took their medications at least once during each consecutive day. If on a given day the medication was not taken there can be two reasons: the patient had previously discontinued treatment (nonpersistence) or the patient was still engaged with the dosing regimen but neglected to take a dose on that particular day (non-execution).

We used Kaplan-Meier curves to display persistence over time. Persistence was censored if there was no evidence of discontinuation in treatment at the end of the observation period.

Quality of execution should be interpreted according to the pharmacokinetic/pharmacodynamic properties of each drug in question. We identified any pattern of dosing histories that could jeopardise efficacy of treatment and investigated its prevalence in the population. We focused on omissions of dose on a single day and two consecutive days and drug holidays, defined as a sequence of at least three consecutive days without taking the drug. We defined omission of a dose on a single day as an interval between subsequent doses of over 30 hours (that is, we arbitrarily added six hours tolerance to the prescribed 24 hour interval between sequential doses). A similar rule was used to define omission of more than one day. We also estimated frequencies of single or sequential dose omissions within and between patients. Logistic models for longitudinal binary data were used to test for potential changes in the daily probability of drug intake over time: days of the week and months of the year.
Finally, we investigated the relation between execution and persistence by plotting Kaplan-Meier estimates of persistence for different strata of execution, formally tested with a Cox's proportional hazards model. All statistical tests were considered significant at the $5 \%$ level.

\section{RESULTS}

The database contained dosing histories of 4783 patients prescribed with once a day antihypertensive drug treatments. They came from 21 phase IV clinical studies, ranging in length between 30 and 330 days and involving 43 different antihypertensive drugs, including angiotensin II receptor blockers $(\mathrm{n}=2088)$, calcium channel blockers $(\mathrm{n}=937)$, angiotensin converting enzyme inhibitors $(\mathrm{n}=665), \beta$ blockers $(\mathrm{n}=195)$, and diuretics $(\mathrm{n}=155)$. The final dataset included data on 478630 days of dosing history.

Figure 1 shows samples of chronology plots from four patients with different patterns of adherence. The first patient perfectly executed the dosing regimen and was persistent during an observation period of 60 days. The second patient also executed the dosing regimen reasonably well but discontinued treatment after 60 days, although he was expected to continue at least until the end of the observation period of 280 days. The third patient executed the regimen poorly, several doses were missed, and a long drug holiday occurred. Finally, at day 180, the patient discontinued treatment. The fourth patient took most of the scheduled doses and persisted for 307 days, but the time he took the drug varied greatly.

Figure 2 shows the results when we applied the definitions described in the methods. The persistence line represents the decline, over time since the start of treatment, in the proportion of patients who are still engaged with the dosing regimen. By the end of one year, almost half of the patients who were prescribed an antihypertensive medication have stopped taking the treatment, despite a dosing regimen of indefinitely long, continuous dosing specified in the protocol. The initial abrupt small drop in the persistence curve represents the proportion of patients who never engaged with the dosing regimen. These occurrences of non-acceptance represent $2 \%$ of the studied popula-

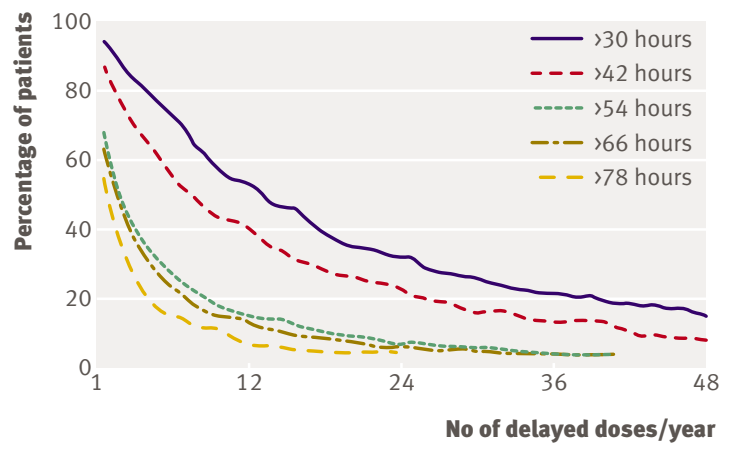

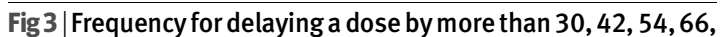
and 78 hours within and between patients 
tion. After the sharp small initial drop of nonacceptance, the persistence curve decreases gradually but progressively over time. For example, at day 200, $35 \%$ of the patients have already stopped the treatment - that is, $65 \%$ persist with treatment. On, for example, the 200th day, among patients still engaged with the dosing regimen, $10 \%$ did not take their medication (non-execution). The percentage of the patients comprising the inception cohort who took their dose on day 200 is thus $58 \%(90 \% \times 65 \%)$, constituting a measure of overall shortfall in drug intake. In terms of crude percentages, non-execution of the dosing regimen thus created a shortfall in drug intake that is an order of magnitude smaller than the shortfall created by early discontinuation/short persistence.

On each day of treatment, about $10 \%$ of scheduled doses were omitted. The breakdown of these omissions is as follows: $42 \%$ were omission of a single day's dose; $15 \%$ were omission of one or two consecutive days' doses, and $43 \%$ were one of a longer, multi-day sequence of omitted doses (three or more days). Figure 3 shows the frequency of missing doses within and between patients. Almost $95 \%$ of the patients missed a single dose (that is, had an interval of over 30 hours since the last taken dose) at least once a year. Half of the patients missed a single day's dose at a rate of one a month (12 a year); $48 \%$ of the patients took a drug holiday ( $>78$ hours) at least once a year; and 13\% had bi-monthly (six a year) drug holidays.

We identified periodic patterns in execution. There was a small but definite seasonal trend with a $2 \%$ reduction in execution between April and September $(\mathrm{P}<0.001)$. In a third of the patients we identified one or more days of the week on which errors occurred at exceptionally high rates: weekend doses were more likely to be missed than weekday doses (odds ratio 1.13, $95 \%$ confidence interval 1.12 to 1.15 ), but occasionally some patients had a particular weekday in which dosing errors were especially clustered. Finally, we classified patients as morning takers $(n=4149)$ if they took more than $75 \%$ of doses between 3 am and $3 \mathrm{pm}$, or evening takers $(\mathrm{n}=283)$ if they took more than $75 \%$ of doses between $3 \mathrm{pm}$ and $3 \mathrm{am}$, and "variable" $(\mathrm{n}=257)$ if they could not be classified as either. Figure 4 shows the proportion of missed doses by day of the week for each category of takers. Morning takers were more likely to take treatment correctly than evening takers $(1.38,1.36$ to 1.41$)$ and evening takers were more likely to take treatment correctly than variable takers $(1.48,1.45$ to 1.52). Sunday morning was when morning takers missed most doses; Saturday evening was when evening takers missed most doses.

Figure 5 displays the estimates of persistence stratified by the degree of execution, clearly showing that the better the execution, the longer the persistence. The likelihood that a patient would discontinue treatment early was related to the quality of his/her daily execution of the dosing regimen (hazard ratio 0.84 for $10 \%$ increase in execution; $95 \%$ confidence interval 0.81 to 0.87 ).

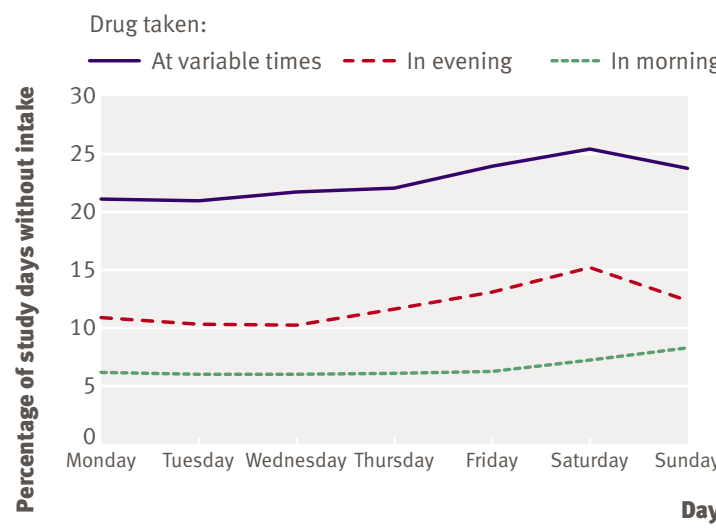

Fig 4 | Proportion of days without drug intake by day of week for each category of takers: morning, evening, or variable

\section{DISCUSSION}

This retrospective analysis of dosing histories of patients prescribed once a day antihypertensive drugs showed that non-persistence is the leading problem with adherence: half of the patients stopped treatment within a year; $48 \%$ had at least one drug holiday a year and almost $95 \%$ of them missed at least a single dose a year; failure to take a dose was more common at weekends; the better a patient executed the drug regimen, the more likely he or she was to persist with the prescribed dosing regimen; morning dosing was more likely to be executed properly than evening dosing; and there was a small seasonal pattern of drug adherence.

\section{Extent of the problem}

Early discontinuation of treatment is a major problem with long term antihypertensive treatment. According to our analysis, we can expect about half of the patients to stop treatment within one year, despite having been prescribed longer term treatment. In this respect, the degree of persistence estimated from the database confirms the results obtained from the timing of refills in large prescription databases. Indeed, in a population of 22918 patients, Caro et al estimated the six month persistence to be $68 \%,{ }^{10}$ hardly different from the present estimation of $66 \%$. Other estimates of persistence at one year are around $48 \%$ in 21723 patients $^{20}$ and $51 \%$ in 82824 patients. $^{21}$

Large prescription databases, derived from timing of prescription refills, constitute a form of validation for our database, but refill data do not reveal the times when dosing errors occurred nor can they serve as a basis for proactive intervention to prevent early discontinuation because these deviations from the prescribed dosing regimens are revealed only months after the fact by refill audits.

\section{Electronically compiled drug dosing history}

Electronic monitoring of medication events-based on electronic detection of opening a container-is of course an indirect method of estimating when and how much drug is ingested. A patient could open the container but not take the drug or take a different dose 


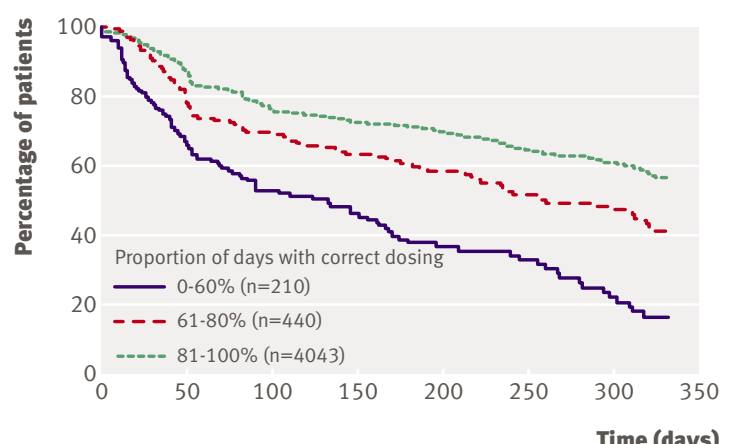

$\overline{\text { Fig } 5 \text { | Kaplan-Meier persistence curves stratified by proportion }}$ of prescribed drug taken

than the one prescribed. The key operational question is how often does this happen? The definitive test is to use sequences of medication events as input to a patient specific pharmacokinetic model of the relation between dose ingested and the subsequent time course of concentration of drug in plasma, comparing projected and periodically measured concentrations of drug in plasma. Results of such investigations, one of which continued throughout one year, ${ }^{17}$ confirmed the validity of using medication event monitors, ${ }^{22-24}$ signifying that mismatches between medication events and actual dosing were too rare to have created substantial differences between projected and actual concentrations of drug in plasma. The reliable projection of drug concentrations in plasma from drug dosing histories is the ideal test of any method that purports to compile drug dosing histories in ambulatory patients.

\section{Strengths and weaknesses}

The limitations of this study are those inherent in retrospective studies of small diverse populations where data have been collected in a clinical context. The studies included in this analysis are heterogeneous and represent an array of objectives, drugs used, and lengths of follow-up. They were, however, all phase IV studies of recently introduced antihypertensive agents,

\section{WHAT IS ALREADY KNOWN ON THIS TOPIC}

Poor adherence to antihypertensive treatment is a major therapeutic challenge and contributes to the lack of adequate control in more than two thirds of patients with hypertension

Although "adherence" seems a simple construct, often reduced to a percentage of prescribed doses taken, electronically compiled dosing histories reveal variably long intervals between doses and variably short durations of treatment

\section{WHAT THIS STUDY ADDS}

The principal modes of non-adherence are quitting treatment early, and, before treatment ends, suboptimal execution of the once a day dosing regimen, with intervals between doses of three or more days

About half of patients quit treatment within the first year; they made many more errors in execution of the dosing regimen than those who continued treatment

Patients who omit sequential doses are at highest risk of quitting early and should be targeted and proactively re-motivated to continue treatment in general designed to show each agent's action in settings representative of expected clinical use in patients with uncomplicated hypertension. It seems reasonable to assume that the aggregated data reflect a representative view of patterns of drug adherence in such patients.

The various trials were carried out according to then prevailing standards for design and interpretation. The data on dosing history were automatically compiled for each patient in a standardised database. Inclusion of each patient's demographic characteristics, however, was optional and was present for only a minority of patients: information on sex was available for 1062 patients (22\%) and on age for only 163 patients (3.4\%). In these subsamples, the mean age was 59 years (SD 13 years) and 58\% were men. These estimates are similar to reported values ${ }^{25}$ and consistent with the hypothesis that the studied population is probably representative of patients treated for uncomplicated hypertension.

This database probably gives a reasonable approximation of the various temporal patterns in intake of antihypertensive drugs in patients with uncomplicated hypertension: within day (morning or afternoon) differences, day of week differences, a relation between sequential dose omissions and early discontinuation, and seasonal differences. Thus, ongoing information on the quality of execution might signify impending early discontinuation, possibly allowing an opportunity to intervene early to prevent treatment interruption. A similar earlier observation was made in patients with epilepsy in whom a disorganisation of execution often preceded a long drug holiday and could be prevented by a pharmacist's telephone inter vention. $^{26}$

\section{Relevance of results}

These results are clinically important in several ways. Firstly, they show the various dosing patterns that could be potential targets for the management of long term drug therapy. These results also emphasise the importance of correctly identifying whether the mode of non-adherence is poor execution or non-persistence because the necessary intervention will differ substantially. Patients who execute poorly need help in integrating their daily dosing into their routine, whereas patients who are at risk of imminent discontinuation need reinforcement and re-motivation to continue with the treatment. Behavioural approaches have been proposed to improve drug adherence, ${ }^{7}$ but our data suggest the value of a management oriented approach to avoiding early discontinuation by remotivation and improvement of the quality of regimen execution by helping patients to integrate their dosing into daily routines..$^{2728}$

These findings have implications for practical clinical management of treatment of hypertension, which are possibly also applicable to other long term drug treatments. Whenever possible drugs should be taken in the morning, and patients and care givers should identify barriers to adherence during weekends 
and other leisure times. One aspect of this effort is the use, when possible, of antihypertensive drugs that have the ability to sustain full pharmacological action for one to two dosing cycles after a dose has been missed, and perhaps longer. Antihypertensive drugs with this degree of "forgiveness" 2930 will compensate for most dose omissions but not for short persistence.

These data suggest the utility of a proactive, measurement guided management programme that is focused on ensuring that patients adhere as closely as possible to the prescribed dosing regimens for antihypertensive drugs. Several studies have shown that effective management of medication, when guided by reliable data on dosing history, can enhance daily execution of and long persistence with the prescribed drug dosing regimen. ${ }^{3132}$ Both are obvious prerequisites for long term control of blood pressure.

Lastly, the low persistence and the frequency of missed doses in these studies questions the validity of drug trials in which neither persistence nor execution are measured and taken into account. Clinical results should be related to the doses taken and not to an assumed $100 \%$ persistence and execution, as is often done when reliable data on dosing history are unavailable. Moreover, so called "safety data" are unreliable when the exposure to the drug of many of the studied patients is low or non-existent and easily camouflaged by, for example, discarding untaken doses, denying that doses were missed, and false diary entries.

Contributors: BV conceived and designed the study, prepared the first draft, and is guarantor. BV and PK acquired and analysed the data. All authors participated in interpreting the results of the analysis. All authors reviewed and drafted the subsequent versions of the manuscript. Funding: Novartis Pharma, Switzerland.

Competing interests: $\mathrm{JU}$ is a shareholder of Aardex, the company that manufactures the MEMS monitors.

Ethical approval: Not required.

Provenance and peer review: Not commissioned; externally peer reviewed.

1 MacMahon S, Peto R, Cutler J, Collins R, Sorlie P, Neaton J, et al. Blood pressure, stroke and coronary heart disease. Part 1. Prolonged differences in blood pressure: prospective observational studies corrected for the regression dilution bias. Lancet 1990;335:765-74.

2 Canto JG, Iskandrian AE. Major risk factors for cardiovascular disease: Debunking the "only 50\%" myth. JAMA 2003;290:947-9.

3 The sixth report of the joint national committee on prevention, detection, evaluation, and treatment of high blood pressure. Arch Int Med 1997;157:2413-56.

4 Waeber B, Burnier M, Brunner HR. Compliance with antihypertensive treatment. Clin Exp Hypertens 1999;21:973-85.

5 Burnier M. Medication adherence and persistence as the cornerstone of effective antihypertensive therapy. Am J Hypertens 2006;19:1190-6.

6 Hyre AD, Krousel-Wood MA, Muntner P, Kawasaki L, DeSalvo KB. Prevalence and predictors of poor antihypertensive medication adherence in an urban health clinic setting. J Clin Hypertens 2007;9:179-86.
7 Schroeder K, Fahey T, Ebrahim S. How can we improve adherence to blood pressure-lowering medication in ambulatory care? Arch Intern Med 2004;164:722-32.

8 PullarT, Kumar S, Feely M. Compliance in clinical trials. Ann Rheum Dis 1989;48:871-5.

9 Rudd P, Ahmed S, Zachary V, Barton C, Bonduelle D. Improved compliance measures: applications in an ambulatory hypertensive drug trial. J Clin Pharmacol Ther 1990;48:676-85.

10 Caro JJ, Speckmann JL, Salas M, Raggio G, Jackson JD. Effect of initial drug choice on persistence with antihypertensive therapy: the importance of actual practice data. CMAI 1999;160:41-6.

11 Halpern MT, Khan ZM, Schmier JK, Burnier M, Caro JJ, Cramer J, et al. Recommendations for evaluating compliance and persistence with hypertension therapy using retrospective data. Hypertension 2006;47:1039-48

12 Feinstein AR. On white-coat effects and the electronic monitoring of compliance. Arch Intern Med 1990;150:1377-8.

13 Cramer JA, Scheyer RD, Mattson RH. Compliance declines between clinic visits. Arch Intern Med 1990;150:1509-10.

14 Duesing R. Adverse events, compliance, and changes in therapy. Curr Hypertens Rep 2001;3:488-92.

15 Feldman SR, Camacho FT, Krejci-Manwaring J, Carroll CL, Balkrishnan R. Adherence to topical therapy increases around the time of office visits. I Am Acad Dermatol 2007;57:81-3.

16 Ebrahim S. Detection, adherence and control of hypertension for the prevention of stroke: a systematic review. Health Technol Assess 1998;2:1-78.

17 Vrijens B, Tousset E, Rode R, Bertz R, Mayer S, Urquhart J. Successful projection of the time course of drug concentration in plasma during a 1 -year period from electronically compiled dosing-time data used as input to individually parameterized pharmacokinetic models. / Clin Pharmacol 2005;45:461-7.

18 Urquhart J, Vrijens B. New findings about patient adherence to prescribed drug dosing regimens: an introduction to pharmionics. Eur J Hospital Pharm Sci 2005;11:103-6.

19 Vrijens B, Goetghebeur E. Comparing compliance patterns between randomized treatments. Control Clin Trials 1997;18:187-203.

20 Bloom BS. Daily regimen and compliance with treatment. BMJ 2001;323:647.

21 Morgan SG, Yan L. Persistence with hypertension treatment among community-dwelling BC seniors. Can I Clin Pharmacol 2004;11:e267-73.

22 Rubio A, Cox C, Weintraub M. Prediction of diltiazem plasma concentration curves from limited measurements using compliance data. Clin Pharmacokinet 1992;22:238-46.

23 Girard P, Sheiner LB, Kastrissios H, Blaschke TF. Do we need full compliance data for population pharmacokinetics analysis? J Pharmacokinet Biopharm 1996;24:265-82.

24 Vrijens B, Goetghebeur E. The impact of compliance in pharmacokinetic studies. Stat Meth Med Res 1999;8:247-62.

25 Hyman DJ, Pavlik VN. Characteristics of patients with uncontrolled hypertension in the United States. N Engl J M 2001;345:479-85.

26 Schneider MP, Despland PA, Buclin T, Burnier M. Evaluation of online telemonitoring of drug adherence: a pilot randomised, controlled study in patients with epilepsy. J Inform Tech Healthcare 2003;1:419-35.

27 Rosen MI, Rigsby MO, Salahi JT, Ryan CE, Cramer JA. Electronic monitoring and counseling to improve medication adherence. Behav Res Ther 2004;42:409-22.

28 Cramer JA, Rosenheck R. Enhancing medication compliance for people with serious mental illness. J Nerv Ment Dis 1999;187:53-5.

29 Urquhart J. Patient non-compliance with drug regimens: measurement, clinical correlates, economic impact. Eur Heart 1996;17(suppl A):8-15.

30 Urquhart J. Pharmacodynamics of variable patient compliance: implications for pharmaceutical value. Adv Drug Deliv Rev 1998;33:207-19.

31 Burnier M, Schneider MP, Chiolero A, Stuci CL, Brunner HR. Electronic compliance monitoring in resistant hypertension: the basis for rational therapeutic decisions. J Hypertens 2001;19:335-41.

32 Vrijens B, Belmans A, Matthys K, de Klerk E, Lesaffre E. Effect of patien intervention and compliance-enhancing pharmaceutical care on adherence with atorvastatin. Pharmacoepidemiol Drug Saf 2006;15:115-21.

Accepted: 14 April 2008 